\title{
Hepatic Fat Content and Liver Enzymes Are Associated with Circulating Free and Protein-Bound Advanced Glycation End Products, Which Are Associated with Low-Grade Inflammation: The CODAM Study
}

\author{
Mitchell Bijnen (iD), ${ }^{1,2}$ Marleen M. J. van Greevenbroek, ${ }^{1,2}$ Carla J. H. van der Kallen, \\ Jean L. Scheijen $\mathbb{D}^{1,2}$ Marjo P. H. van de Waarenburg, ${ }^{1,2}$ Coen D. A. Stehouwer $\mathbb{D}^{1,2}$ \\ Kristiaan Wouters, ${ }^{1,2}$ and Casper G. Schalkwijk (iD ${ }^{1,2}$ \\ ${ }^{1}$ Department of Internal Medicine, MUMC, Maastricht, Netherlands \\ ${ }^{2}$ CARIM, MUMC, Maastricht, Netherlands
}

Correspondence should be addressed to Casper G. Schalkwijk; c.schalkwijk@maastrichtuniversity.nl

Received 20 December 2018; Revised 28 February 2019; Accepted 5 March 2019; Published 14 May 2019

Academic Editor: Mark Yorek

Copyright (C) 2019 Mitchell Bijnen et al. This is an open access article distributed under the Creative Commons Attribution License, which permits unrestricted use, distribution, and reproduction in any medium, provided the original work is properly cited.

Advanced glycation end products (AGEs) accumulate in fatty livers and may contribute to low-grade inflammation (LGI), potentially via their receptor, RAGE. It is unknown if the AGE accumulation in fatty livers results in elevated circulating AGEs. In a cohort study, we investigated the association of liver fat and hepatocellular damage with circulating AGEs and soluble RAGE (sRAGE) and subsequently the association of circulating AGEs and sRAGE with LGI. Cross-sectional associations of liver fat percentage (eLF\%; ln-transformed) and liver enzymes (LE score; standardized) with circulating AGEs (free CML, CEL, and MG-H1 in $\mathrm{nM}$ and protein-bound CML, CEL, and pentosidine in nmol/mmol lysine; ln-transformed) and sRAGE (pg/ml, ln-transformed) and additionally of AGEs and sRAGE with LGI (standardized) were determined by multiple linear regression. eLF\% was positively associated with circulating free CEL $(\beta=0.090 ; 95 \%$ CI $0.041 ; 0.139)$ but inversely with protein-bound CML $(\beta=-0.071 ; 95 \%$ CI $-0.108 ;-0.034)$. Similarly, the LE score was positively associated with free CML $(\beta=0.044 ; 95 \%$ CI $0.012 ; 0.076)$ and $\operatorname{CEL}(\beta=0.040 ; 95 \%$ CI $0.009 ; 0.072)$ but inversely with protein-bound CML $(\beta=-0.037 ; 95 \%$ CI $-0.060 ;-0.013)$. Free CML $(\beta=0.297 ; 95 \%$ CI $0.049 ; 0.545)$ was positively associated with LGI, while protein-bound CML $(\beta=-0.547 ; 95 \%$ CI $-0.888 ;-0.207)$ was inversely associated, although this association was absent after adjustment for BMI. eLF\% and LE score were not associated with sRAGE and sRAGE not with LGI after adjustment for BMI. Liver fat and enzymes were positively associated with circulating free AGEs, which were associated with LGI. In contrast, inverse relations were observed of liver fat and enzymes with circulating protein-bound AGEs and of protein-bound AGEs with LGI. These data suggest that hepatic steatosis and inflammation affect the formation and degradation of hepatic protein-bound AGEs resulting in elevated circulating free AGE levels. These alterations in AGE levels might influence LGI, but this is likely independent of RAGE.

\section{Introduction}

Nonalcoholic fatty liver disease (NAFLD) is a spectrum of liver abnormalities ranging from steatosis (fatty liver) to nonalcoholic steatohepatitis (NASH), fibrosis, and potentially even cirrhosis. NASH is characterized by both steatosis and inflammation, of which the latter causes hepatocellular injury and over time irreversible liver injury [1]. Moreover, NASH is associated with cardiovascular disease (CVD), seemingly due to hepatic inflammation considering that long-term survival of CVD-related diseases is lower in NASH patients than in NAFLD patients with steatosis only [2,3]. Therefore, it is highly relevant to investigate the causes of hepatic inflammation and how these might affect CVD risk. 
Fat accumulation in the liver, i.e., steatosis, can cause oxidative stress, increased lipid peroxidation, and release of inflammatory cytokines $[4,5]$. Higher levels of oxidative stress and lipid peroxidation, accompanied by inflammationinduced elevated metabolic rate, stimulate the formation of advanced glycation end products (AGEs) [6, 7]. These sugar-modified proteins are capable of disturbing intracellular protein function, cross-linking extracellular matrix (ECM) proteins, and activating the receptor of advanced glycation end products, RAGE [8]. AGEs can be present in both the free (glycated free amino acids) and proteinbound (glycated amino acids within a protein) form. Considering that many of the amino acids in the circulation are derived from degraded proteins, free AGEs are likely derived from degradation of protein-bound AGEs [9]. Major AGEs include $\mathrm{N}^{\varepsilon}$-(carboxymethyl)lysine (CML), $\mathrm{N}^{\varepsilon}$-(1-carboxyethyl)lysine (CEL), $\mathrm{N}^{\delta}$-(5-hydro-5-methyl-4imidazolon-2-yl)-ornithine (MG-H1), and pentosidine. Binding and stimulation of the receptor for advanced glycation end products (RAGE) by protein-bound CML (PB-CML), protein-bound CEL (PB-CEL), and MG-H1 can increase oxidative stress and upregulate transcription of NF- $\kappa \mathrm{B}-$ dependent genes, i.e., inflammatory genes [10-12]. Furthermore, our group demonstrated that RAGE can trap the protein-bound form of AGEs in obese adipose tissue thereby inducing adipose tissue inflammation [13]. Besides as a cell membrane-bound form that stimulates inflammation, RAGE can also be present as soluble RAGE (sRAGE) in plasma due to alternative splicing (i.e., esRAGE) or cleavage of the already cell-bound form $[14,15]$. The functional role of sRAGE has not yet been fully elucidated, but it is thought to act as a decoy receptor for its ligands [15]. Alternatively, it might simply be an indication of RAGE activity as it has been associated with CVD and diabetes $[16,17]$.

We previously showed that CML accumulates in human fatty livers. This accumulation was more pronounced in livers with more severe steatosis and inflammation [6]. In addition, we demonstrated that PB-CML exerts proinflammatory effects on hepatocytes via RAGE, resulting in the production of inflammatory cytokines [6]. We now hypothesize that AGEs that accumulate in the fatty liver are released into the circulation and also activate RAGE in the liver, thereby contributing to low-grade inflammation (LGI). In a human cohort study, we investigated the association of (1) liver fat and liver enzymes with circulating AGEs or sRAGE and (2) the association of circulating AGEs and sRAGE with LGI.

\section{Materials and Methods}

2.1. Subjects and Study Design. Cohort on Diabetes and Atherosclerosis Maastricht (CODAM) is a cohort study of 574 subjects, selected based on a moderately increased risk of cardiometabolic disease from a large cohort in the general population as previously described [18]. The current crosssectional analyses were performed in 505 subjects as subjects with missing data (either main dependent (outcome) or main independent variable or important covariate) were excluded. The study protocol was approved by the Medical Ethical Committee of the Maastricht University Medical Centre, in line with the ethical guidelines of the 1975 Declaration of Helsinki, and all subjects gave written informed consent.

2.2. Liver Fat Estimation. Liver fat percentage (eLF\%) was estimated using a magnetic resonance spectroscopy- (MRS-) validated equation developed by Kotronen et al. [19]. Briefly, liver fat content is estimated based on liver enzymes (ASAT (aspartate amino transferase) and ASAT/alanine transaminase (ALAT) ratio), fasting insulin levels, presence of the metabolic syndrome, and presence of type 2 diabetes. In addition, the fatty liver index (FLI) as described by Bedogni et al. was used. This estimation is based on an algorithm using BMI, waist circumference, triglycerides, and gammaglutamyl transferase (GGT) levels and was validated using ultrasound and MRS [20, 21].

2.3. Liver Enzymes and Inflammatory Markers. ASAT, ALAT, and GGT were measured in EDTA plasma as previously described [22]. Plasma markers of low-grade inflammation (IL-6, IL-8, TNF- $\alpha$, CRP, SAA, and sICAM1) were measured using an MSD multiplex assay.

2.4. Measurement of Advanced Glycation End Products. Free CML, CEL, and MG-H1 and PB-CML, PB-CEL, and protein-bound pentosidine (PB-pentosidine) were analysed in EDTA plasma by ultraperformance liquid chromatography tandem mass spectrometry (UPLC MS/MS). Details of the measurement of plasma AGEs have been previously described [23].

2.5. Measurement of sRAGE. sRAGE (esRAGE and shed forms of RAGE) levels were determined in EDTA plasma using a human RAGE quantikine ELISA (R\&D Systems) according to the manufacturer's instructions. Intra- and interassay coefficients of variation were 7.6 and $2.6 \%$, respectively.

2.6. Other Covariates. Body mass index (BMI), smoking status, mean alcohol consumption, medication use, prior CVD, and glomerular filtration rate (eGFR; estimated using the short Modification of Diet in Renal Disease equation) were determined as previously reported [22].

2.7. Statistical Analysis. Study population characteristics were compared across tertiles of liver fat percentage using standard one-way ANOVA with a Bonferroni post hoc test for normally distributed variables, Kruskal-Wallis one-way ANOVA with a Bonferroni post hoc test for non-normally distributed variables, and a Chi-square test with a Bonferroni correction for categorical variables. A combined liver enzyme (LE) or low-grade inflammation (LGI) score was calculated as previously described [22]. In brief, all LEs or LGI plasma markers were ln-transformed to obtain a normal distribution. For each individual, these ln-transformed values were transformed into $Z$-scores (i.e., standardized values). Next, the three standardized LEs and six inflammation markers, respectively, were averaged to obtain a composite score, which was again standardized. The resulting LE score and LGI score were used in subsequent analyses. All AGEs, eLF\%, and sRAGE were also ln-transformed prior to analyses. FLI was standardized before analyses. 
The cross-sectional associations of liver fat and liver enzymes (main independent variables) with AGEs and sRAGE (outcomes) and additionally of AGEs and sRAGE (main independent variables) with LGI (outcome) were examined with the use of multiple linear regression analyses with adjustments for the following potential confounders: age (years), sex (male/female), alcohol consumption (g/day), current smoker $(\mathrm{Y} / \mathrm{N})$, prevalent $\mathrm{CVD}(\mathrm{Y} / \mathrm{N})$, use of medication (glucose-lowering $(\mathrm{Y} / \mathrm{N})$, lipid-modifying $(\mathrm{Y} / \mathrm{N})$, and antihypertensive $(\mathrm{Y} / \mathrm{N}))$, eGFR $\left(\mathrm{ml} / \mathrm{min} / 1.73 \mathrm{~m}^{2}\right)$, and BMI $\left(\mathrm{kg} / \mathrm{m}^{2}\right)$. All linear regression analyses were performed with four different models as follows: model 1: crude model; model 2: adjustment for age and sex; model 3: adjustment for age, sex, alcohol, smoking, CVD, medication, and eGFR; model 4: adjustment for age, sex, alcohol, smoking, CVD, medication, eGFR, and BMI. All statistical analyses were performed using the SPSS for Windows, version 25.0 (IBM Corp.), and all data were considered statistically significant at $P \leq 0.05$.

\section{Results}

3.1. Study Population. In Table 1, the study population is presented according to tertiles of eLF\%. Subjects with more severe steatosis in general had a higher prevalence of type 2 diabetes and CVD and accordingly used more medication. Moreover, individuals in the highest liver fat tertile had the highest BMI, fasting glucose, triglyceride, and HbA1c levels but the lowest HDL level. These worse metabolic characteristics were accompanied by higher levels of low-grade inflammation markers and liver enzymes. PB-pentosidine, PB-CML, and sRAGE were lower, while free CEL was higher in those with the highest amount of liver fat.

3.2. Association of Liver Fat with Free and Protein-Bound $A G E s$ and $s R A G E$. In linear regression analyses, eLF\% was positively associated with free CML $(\beta=0.042 ; 95 \% \mathrm{CI}$ $0.002 ; 0.082)$ and CEL $(\beta=0.096 ; 95 \%$ CI $0.058 ; 0.135)$ but inversely with $\mathrm{PB}$-pentosidine $(\beta=-0.059 ; 95 \%$ CI -0.1 ; -0.018), PB-CML $(\beta=-0.115 ; 95 \%$ CI $-0.143 ;-0.087)$, and sRAGE $(\beta=-0.097 ; 95 \%$ CI $-0.146 ;-0.048)$. Adjustment for age, sex, alcohol consumption, smoking, prior CVD, medication use, and eGFR (model 3) mainly affected the positive association of eLF\% with free CML, which was no longer significant $(\beta=0.041 ; 95 \%$ CI $-0.002 ; 0.084)$. After additional adjustment for BMI (model 4), eLF\% was still positively associated with free CEL and inversely with PB-CML, but the association with free CML, PB-pentosidine, or sRAGE was no longer significant. eLF\% was not associated with PB-CEL or MG-H1 (Table 2). Additional analyses were performed with FLI instead of the eLF\% as a measure of liver fat and yielded comparable results (Suppl. Table 1). However, the positive association between FLI and free CML now remained significant after adjustment of several confounders (model 3), and the inverse association between FLI and PB-pentosidine was also retained after adjustment for BMI (model 4). Of note, adjusting for waist circumference instead of BMI in model 4 did not materially change the results (data not shown).
3.3. Association of Liver Enzymes with Free and ProteinBound AGEs and SRAGE. Next, we investigated the association of the LE score (as a measure of hepatocellular injury) with AGEs and sRAGE (Table 2). The LE score was positively associated with free CML $(\beta=0.030 ; 95 \%$ CI $0 ; 0.060)$ and free CEL $(\beta=0.047 ; 95 \%$ CI $0.018 ; 0.077)$, but an inverse association between the LE score and PB-CML $(\beta=-0.061$; $95 \%$ CI $-0.083 ;-0.039)$ or the LE score and sRAGE ( $\beta=-0.072$; 95\% CI $-0.109 ;-0.034)$ was observed. The positive associations with free CML $(\beta=0.046 ; 95 \%$ CI 0.015 ; $0.077)$ and free CEL $(\beta=0.049 ; 95 \%$ CI $0.019 ; 0.079)$ were stronger after additional adjustment (model 3), while the association with PB-CML $(\beta=-0.054 ; 95 \%$ CI -0.077 ; -0.031) was slightly weaker and the association with sRAGE was no longer significant $(\beta=-0.027$; 95\% CI -0.067; 0.012). The LE score was not associated with PB-CEL or MG-H1. Neither adjustment for BMI (Table 2) nor for waist circumference (data not shown) altered these associations substantially.

3.4. Association of Liver Fat and Liver Enzymes with LGI. In our cohort, we next investigated the associations between liver fat or liver enzymes and the LGI score (Table 3 ). There was a strong association of $\mathrm{eLF} \%(\beta=0.494 ; 95 \%$ CI 0.387 ; $0.601)$ and the LE score $(\beta=0.310 ; 95 \%$ CI 0.226 ; 0.393$)$ with LGI. Additional adjustment did not substantially affect these associations. Moreover, when employing the FLI instead of eLF\% (Suppl. Table 2) or waist circumference instead of BMI (data not shown), the results were highly comparable.

3.5. Association of Free and Protein-Bound AGEs and sRAGE with LGI. AGEs can trigger inflammation via RAGE, potentially contributing to LGI and could thereby explain, at least in part, the association of liver fat and liver enzymes with LGI. Therefore, we investigated the association of AGEs and sRAGE with the LGI score. Free CML $(\beta=0.443 ; 95 \%$ CI $0.194 ; 0.692)$ and free CEL $(\beta=0.359 ; 95 \%$ CI 0.103 ; $0.616)$ were positively associated with LGI, while PB-CML $(\beta=-0.631 ; 95 \%$ CI $-0.965 ;-0.298)$ was inversely associated with this estimate of systemic inflammation. After adjustment for age, sex, alcohol consumption, smoking, prior CVD, medication use, and eGFR (model 3), the positive association between free CEL and LGI was borderline nonsignificant $(\beta=0.259 ; 95 \% \mathrm{CI}-0.006 ; 0.525)$, while the associations of free CML (positive) and PB-CML (inverse) with LGI remained significant. Adjustment for BMI (model 4) strongly attenuated the inverse association between PB-CML and LGI. This association was no longer significant $(\beta=-0.251$; $95 \%$ CI $-0.592 ; 0.090)$, and only the positive association between free CML and LGI remained (Table 4). To examine if free CML indeed contributes to the above-described strong association of eLF\% or LE score with LGI, we performed an additional analysis in which we adjusted that association for free CML. Adjustment for free CML reduced the association between eLF\% and LGI slightly from $\beta=0.429(95 \%$ CI $0.290 ; 0.568)$ to $\beta=0.420(95 \%$ CI $0.281 ; 0.558)$ and the association between the LE score and LGI from $\beta=0.292$ (95\% CI $0.205 ; 0.380)$ to $\beta=$ 0.283 (95\% CI $0.195 ; 0.371)$. No association was observed 


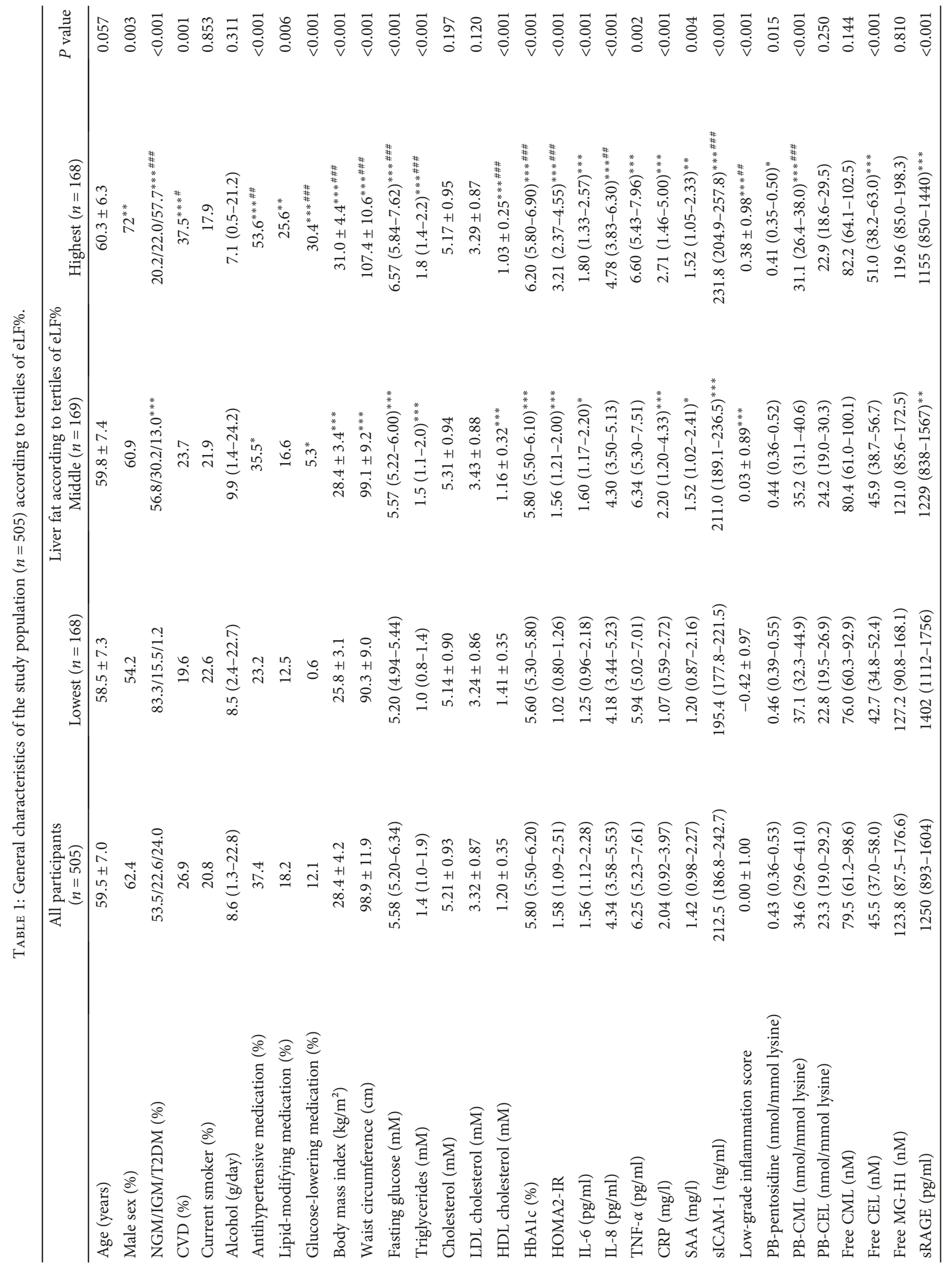




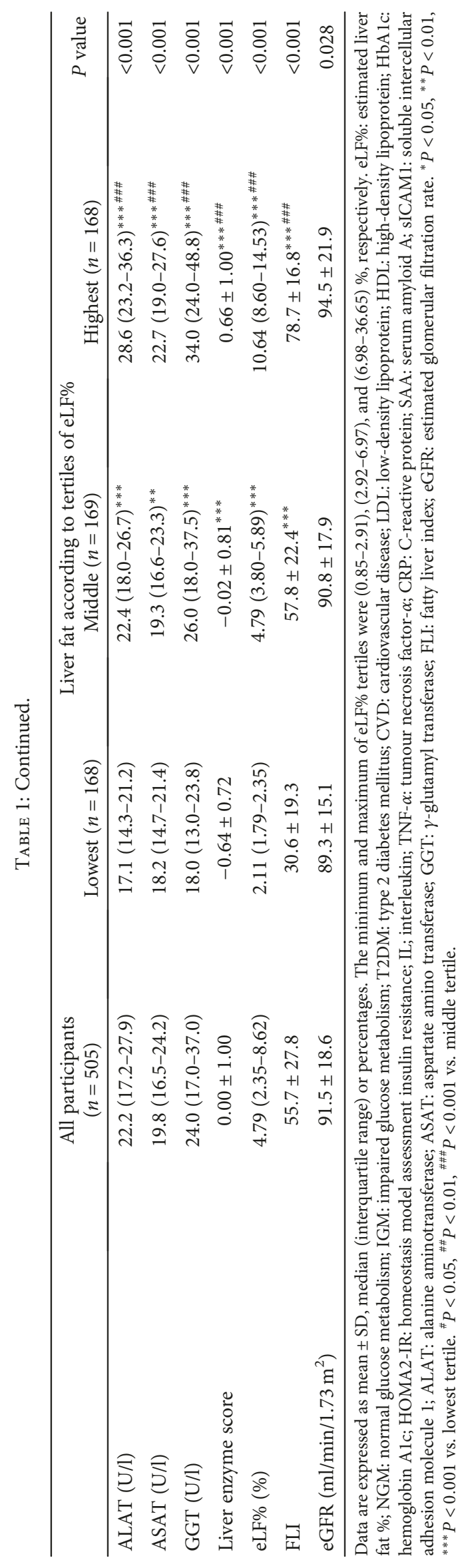


TABLE 2: Cross-sectional associations of liver fat and liver enzymes with free and protein-bound AGEs and sRAGE.

\begin{tabular}{|c|c|c|c|c|c|c|c|c|c|}
\hline \multirow{2}{*}{ Outcome } & \multirow{2}{*}{ Model } & \multicolumn{4}{|c|}{ eLF\% (\%) } & \multicolumn{4}{|c|}{ Liver enzyme score } \\
\hline & & $\beta$ & $95 \% \mathrm{CI}$ & & $P$ value & $\beta$ & $95 \% \mathrm{CI}$ & & $P$ value \\
\hline \multirow{4}{*}{ Free CML (nM) } & 1 & 0.042 & 0.002 & 0.082 & 0.038 & 0.030 & 0.000 & 0.060 & 0.052 \\
\hline & 2 & 0.031 & -0.008 & 0.070 & 0.122 & 0.030 & -0.001 & 0.061 & 0.055 \\
\hline & 3 & 0.041 & -0.002 & 0.084 & 0.061 & 0.046 & 0.015 & 0.077 & 0.004 \\
\hline & 4 & 0.037 & -0.014 & 0.088 & 0.151 & 0.044 & 0.012 & 0.076 & 0.008 \\
\hline \multirow{4}{*}{ Free CEL (nM) } & 1 & 0.096 & 0.058 & 0.135 & $<0.001$ & 0.047 & 0.018 & 0.077 & 0.002 \\
\hline & 2 & 0.083 & 0.045 & 0.121 & $<0.001$ & 0.037 & 0.007 & 0.067 & 0.016 \\
\hline & 3 & 0.095 & 0.054 & 0.136 & $<0.001$ & 0.049 & 0.019 & 0.079 & 0.001 \\
\hline & 4 & 0.090 & 0.041 & 0.139 & $<0.001$ & 0.040 & 0.009 & 0.072 & 0.012 \\
\hline \multirow{4}{*}{ Free MG-H1 (nM) } & 1 & 0.043 & -0.019 & 0.105 & 0.170 & -0.009 & -0.056 & 0.038 & 0.719 \\
\hline & 2 & 0.023 & -0.038 & 0.084 & 0.464 & -0.019 & -0.067 & 0.028 & 0.423 \\
\hline & 3 & 0.024 & -0.042 & 0.090 & 0.481 & 0.006 & -0.042 & 0.053 & 0.820 \\
\hline & 4 & 0.036 & -0.042 & 0.115 & 0.367 & 0.007 & -0.043 & 0.057 & 0.789 \\
\hline \multirow{4}{*}{ PB-pentosidine (nmol/mmol lysine) } & 1 & -0.059 & -0.100 & -0.018 & 0.005 & -0.015 & -0.047 & 0.016 & 0.342 \\
\hline & 2 & -0.076 & -0.117 & -0.035 & $<0.001$ & -0.028 & -0.060 & 0.004 & 0.088 \\
\hline & 3 & -0.102 & -0.146 & -0.058 & $<0.001$ & -0.032 & -0.064 & 0.001 & 0.055 \\
\hline & 4 & -0.051 & -0.102 & 0.001 & 0.055 & -0.007 & -0.040 & 0.026 & 0.676 \\
\hline \multirow{4}{*}{ PB-CML (nmol/mmol lysine) } & 1 & -0.115 & -0.143 & -0.087 & $<0.001$ & -0.061 & -0.083 & -0.039 & $<0.001$ \\
\hline & 2 & -0.122 & -0.150 & -0.093 & $<0.001$ & -0.067 & -0.090 & -0.044 & $<0.001$ \\
\hline & 3 & -0.105 & -0.136 & -0.074 & $<0.001$ & -0.054 & -0.077 & -0.031 & $<0.001$ \\
\hline & 4 & -0.071 & -0.108 & -0.034 & $<0.001$ & -0.037 & -0.060 & -0.013 & 0.002 \\
\hline \multirow{4}{*}{ PB-CEL (nmol/mmol lysine) } & 1 & 0.000 & -0.036 & 0.036 & 0.992 & 0.003 & -0.025 & 0.031 & 0.828 \\
\hline & 2 & -0.002 & -0.039 & 0.035 & 0.911 & -0.002 & -0.030 & 0.027 & 0.919 \\
\hline & 3 & 0.013 & -0.028 & 0.055 & 0.527 & 0.007 & -0.023 & 0.037 & 0.652 \\
\hline & 4 & -0.003 & -0.052 & 0.047 & 0.920 & 0.001 & -0.030 & 0.033 & 0.939 \\
\hline \multirow{4}{*}{ sRAGE (pg/ml) } & 1 & -0.097 & -0.146 & -0.048 & $<0.001$ & -0.072 & -0.109 & -0.034 & $<0.001$ \\
\hline & 2 & -0.076 & -0.125 & -0.028 & 0.002 & -0.040 & -0.078 & -0.002 & 0.039 \\
\hline & 3 & -0.076 & -0.130 & -0.023 & 0.005 & -0.027 & -0.067 & 0.012 & 0.169 \\
\hline & 4 & -0.039 & -0.102 & 0.025 & 0.232 & -0.010 & -0.050 & 0.031 & 0.642 \\
\hline
\end{tabular}

$\beta$-Values are unstandardized regression coefficients and represent the change in AGEs and sRAGE (all ln-transformed) per one unit increase in eLF\% (lntransformed) or the LE score. Model 1: crude model. Model 2: model $1+$ adjustment for age and sex. Model 3: model $2+$ adjustment for alcohol, smoking, CVD, medication, and eGFR. Model 4: model 3 + adjustment for BMI. 95\% CI: 95\% confidence interval.

TABLE 3: Associations of liver fat and liver enzymes with low-grade inflammation.

\begin{tabular}{|c|c|c|c|c|c|c|c|c|c|}
\hline \multirow{2}{*}{ Outcome } & \multirow{2}{*}{ Model } & \multicolumn{4}{|c|}{ eLF\% (\%) } & \multicolumn{4}{|c|}{ Liver enzyme score } \\
\hline & & $\beta$ & $95 \% \mathrm{CI}$ & & $P$ value & $\beta$ & $95 \% \mathrm{CI}$ & & $P$ value \\
\hline \multirow{4}{*}{ LGI } & 1 & 0.494 & 0.387 & 0.601 & $<0.001$ & 0.310 & 0.226 & 0.393 & $<0.001$ \\
\hline & 2 & 0.493 & 0.386 & 0.599 & $<0.001$ & 0.347 & 0.262 & 0.431 & $<0.001$ \\
\hline & 3 & 0.533 & 0.415 & 0.650 & $<0.001$ & 0.356 & 0.270 & 0.442 & $<0.001$ \\
\hline & 4 & 0.429 & 0.290 & 0.568 & $<0.001$ & 0.292 & 0.205 & 0.380 & $<0.001$ \\
\hline
\end{tabular}

$\beta$-Values are unstandardized regression coefficients and represent the change in the LGI score per one unit increase in eLF\% (ln-transformed) or LE score. Model 1: crude model. Model 2: model $1+$ adjustment for age and sex. Model 3: model 2 + adjustment for alcohol, smoking, CVD, medication, and eGFR. Model 4: model 3 + adjustment for BMI. 95\% CI: 95\% confidence interval.

between sRAGE, PB-CEL, PB-pentosidine or MG-H1, and LGI (Table 4). Performing these analyses with adjustment for waist circumference instead of BMI in model 4 did not alter the associations (data not shown).

\section{Discussion}

The present study revealed that liver fat and liver enzymes were positively associated with circulating free AGEs, while 
TABLE 4: Cross-sectional associations of free and protein-bound AGEs and sRAGE with low-grade inflammation.

(a)

\begin{tabular}{cccccccccccccc}
\hline \multirow{2}{*}{ Outcome } & \multirow{2}{*}{ Model } & \multicolumn{4}{c}{ Free CML $(\mathrm{nM})$} & \multicolumn{4}{c}{ Free CEL $(\mathrm{nM})$} & \multicolumn{4}{c}{ Free MG-H1 (nM) } \\
& & $\beta$ & $95 \%$ CI & & $P$ value & $\beta$ & $95 \%$ CI & & $P$ value & $\beta$ & $95 \%$ CI & $P$ value \\
\hline \multirow{4}{*}{ LGI } & 1 & 0.443 & 0.194 & 0.692 & $\mathbf{0 . 0 0 1}$ & 0.359 & 0.103 & 0.616 & $\mathbf{0 . 0 0 6}$ & 0.204 & 0.014 & 0.041 & 0.366 \\
& 2 & 0.322 & 0.067 & 0.577 & $\mathbf{0 . 0 1 3}$ & 0.282 & 0.023 & 0.541 & $\mathbf{0 . 0 3 3}$ & 0.128 & -0.037 & 0.293 & 0.127 \\
& 3 & 0.344 & 0.086 & 0.603 & $\mathbf{0 . 0 0 9}$ & 0.259 & -0.006 & 0.525 & 0.056 & 0.128 & -0.041 & 0.297 & 0.137 \\
& 4 & 0.297 & 0.049 & 0.545 & $\mathbf{0 . 0 1 9}$ & 0.152 & -0.104 & 0.409 & 0.243 & 0.131 & -0.031 & 0.293 & 0.112 \\
\hline
\end{tabular}

(b)

\begin{tabular}{lccccccccccccc}
\hline \multirow{2}{*}{ Outcome } & \multirow{2}{*}{ Model } & \multicolumn{3}{c}{ PB-CML (nmol/mmol lysine) } & \multicolumn{3}{c}{ PB-CEL (nmol/mmol lysine) } & \multicolumn{4}{c}{ PB-pentosidine (nmol/mmol lysine) } \\
& & $\beta$ & $95 \%$ CI & & $P$ value & $\beta$ & $95 \%$ CI & & $P$ value & $\beta$ & $95 \%$ CI & $P$ value \\
\hline \multirow{4}{*}{ LGI } & 1 & -0.631 & -0.965 & -0.298 & $<\mathbf{0 . 0 0 1}$ & -0.047 & -0.325 & 0.231 & 0.741 & -0.059 & -0.303 & 0.185 & 0.637 \\
& 2 & -0.704 & -1.032 & -0.377 & $<\mathbf{0 . 0 0 1}$ & -0.028 & -0.302 & 0.246 & 0.840 & -0.154 & -0.398 & 0.091 & 0.219 \\
& 3 & -0.547 & -0.888 & -0.207 & $\mathbf{0 . 0 0 2}$ & -0.024 & -0.293 & 0.245 & 0.863 & -0.089 & -0.338 & 0.160 & 0.483 \\
& 4 & -0.251 & -0.592 & 0.090 & 0.149 & -0.078 & -0.335 & 0.180 & 0.553 & 0.120 & -0.125 & 0.365 & 0.337 \\
\hline
\end{tabular}

(c)

\begin{tabular}{|c|c|c|c|c|c|}
\hline \multirow{2}{*}{ Outcome } & \multirow{2}{*}{ Model } & \multicolumn{4}{|c|}{ sRAGE $(\mathrm{pg} / \mathrm{ml})$} \\
\hline & & $\beta$ & $95 \% \mathrm{CI}$ & & $P$ value \\
\hline \multirow{4}{*}{ LGI } & 1 & 0.048 & -0.154 & 0.251 & 0.641 \\
\hline & 2 & 0.019 & -0.189 & 0.226 & 0.858 \\
\hline & 3 & 0.037 & -0.170 & 0.244 & 0.725 \\
\hline & 4 & 0.143 & -0.057 & 0.342 & 0.161 \\
\hline
\end{tabular}

$\beta$-Values are unstandardized regression coefficients and represent the change in the LGI score per one unit increase in AGEs or sRAGE (all ln-transformed). Model 1: crude model. Model 2: model $1+$ adjustment for age and sex. Model 3: model $2+$ adjustment for alcohol, smoking, CVD, medication, and eGFR. Model 4: model 3 + adjustment for BMI. 95\% CI: 95\% confidence interval.

inverse associations were observed with circulating proteinbound AGEs. In addition, free AGEs were positively associated with LGI, but an inverse association was determined between protein-bound AGEs and LGI, albeit this latter association was explained by BMI. Lastly, there was no association observed of liver fat and liver enzymes with sRAGE or between sRAGE and LGI after adjustment for BMI.

We demonstrated that a higher hepatic fat content, a hallmark of NAFLD, is associated with AGEs and sRAGE, but a key role is played by obesity and adipose tissue as adjustment for BMI attenuated these associations. However, even after adjustment for this potential confounder, a positive association between liver fat and free AGEs (only CEL and not CML) and an inverse association between liver fat and protein-bound AGEs (pentosidine and $\mathrm{PB}-\mathrm{CML}$ ) was still observed. Lipid peroxidation contributes to CEL formation making its generation likely in metabolically active and lipid-rich environments such as the fatty liver [7]. Indeed, levels of other lipid peroxidation products (e.g. malondialdehyde and 8-isoprostane) are elevated in the circulation of NAFLD patients [24, 25]. Moreover, hepatic steatosis was shown to lead to lipid peroxidation in mice [26]. In line with our data, Palma-Duran et al. recently reported elevated levels of circulating CEL and not CML in NAFLD patients in a case-control study [27].
The inverse relationship between hepatic steatosis and circulating protein-bound AGEs might suggest either trapping of AGEs in the liver or an elevated breakdown of (modified) proteins in fatty livers. We previously reported trapping of $\mathrm{PB}-\mathrm{CML}$ in adipose tissue of obese mice and reduced levels of circulating PB-CML in obese subjects [13]. In the liver, we also observed trapping of CML-modified albumin, although the amount of trapped PB-CML in the liver was not compared between healthy and steatotic livers [13]. Therefore, it is possible that a higher amount of PB-CML is trapped in fatty livers, which could explain the inverse direction of the association between liver fat and circulating PB-CML. Another explanation could be alterations in protein metabolism, specifically an enhanced protein breakdown, in NAFLD. A recent study indeed reported elevated levels of plasma amino acids, including leucine, a marker of protein breakdown, in NAFLD patients [28, 29]. This could also explain the inverse relationship between liver fat and pentosidine. Pentosidine is formed through the slow Maillard reaction and therefore mainly affects long-living proteins, which are typically ECM components such as collagen [30]. In contrast to enhanced protein breakdown, Munsterman et al. reported inhibition of hepatic ECM degradation by hepatic stellate cells in NAFLD [31]. Reduced ECM degradation could explain the observed lower circulating pentosidine levels as 
less modified proteins are released from the ECM, albeit Munsterman et al. reported this in a more advanced stage of NAFLD [31].

We found a strong inverse association between liver fat and sRAGE, but this association was largely dependent on BMI. Several studies have shown that obesity is a strong determinant of sRAGE levels explaining why the association is no longer present after adjustment for this confounder [32, 33]. In accordance with our data, Palma-Duran et al. reported a decrease of sRAGE in NAFLD patients [27]. Interestingly, this difference between cases and controls in their study was observed despite matching for BMI. However, their study used diagnosed NAFLD patients in whom the liver status might be worse than our population. Possibly, sRAGE levels are influenced more by the liver and independently from the obese adipose tissue in patients with worse NALFD.

Our study implies that hepatic injury, represented by liver enzyme levels, is associated with higher levels of circulating free AGEs (CML and CEL), but lower levels of protein-bound AGEs (PB-CML). These findings are in agreement with our previous study revealing elevated levels of CML in livers with more severe steatosis and inflammation, which causes liver injury [6]. Furthermore, hepatic inflammation is accompanied by oxidative stress given that hepatic immune cells are important producers of reactive oxygen species (ROS) [34]. High oxidative stress causes lipid peroxidation and contributes to AGE formation, mainly CML and CEL $[7,35]$.

The association of hepatocellular injury with elevated circulating free CML, but lower circulating PB-CML, suggests enhanced breakdown of proteins by liver injury and the accompanying hepatic inflammation. As previously described, protein degradation is enhanced in NAFLD patients [29]. Moreover, circulating levels of free amino acids correlated strongly with liver enzymes and even hepatic injury and inflammation [29]. These findings make it likely that more intrahepatic PB-CML is degraded to circulating free CML in subjects with more hepatocellular injury.

Our results also show an association of liver fat and liver injury with LGI corroborating that liver disease might contribute to elevated inflammatory cytokines and thereby maintenance of low-grade inflammation $[36,37]$.

The observed positive association of free CML and CEL with LGI suggests that these plasma-free AGEs might influence LGI. However, the association of CEL with LGI disappeared after adjustment for BMI implying that this association depends more on adiposity and the adipose tissue inflammation that accompanies it. Free CML remained associated with LGI even after adjustment for BMI. However, the very modest effect of additional adjustment for free CML suggested that the strong association of hepatic steatosis and injury with LGI was not mediated by circulating free CML levels. Indeed, it has been described that free CML is unable to stimulate RAGE and cause inflammation [11]. Therefore, any effects on LGI by free CML are likely to be indirect or due to disturbance of intracellular function and not via RAGE [8].

In line with this notion, we observed that sRAGE was not associated with LGI despite its potential role as a decoy receptor for its ligands or as indicator of AGE-RAGE axis activity [15-17]. In contrast, Palma-Duran et al. did report an inverse correlation between sRAGE and either TNF- $\alpha$ and CRP, two components of our LGI score [27]. This discrepancy could be explained by the other markers used to calculate our LGI score as these markers might not correlate well with sRAGE. Indeed, when performing a similar analysis, we observed a significant inverse correlation between sRAGE and CRP as well, but the correlation between sRAGE and TNF- $\alpha$ was not present (data not shown). However, we believe that our LGI score is a representative measure of low-grade inflammation and is preferable over a few single markers. Another explanation for discrepancies can be found in the difference in severity of liver disease between the studies. As previously mentioned, Palma-Duran et al. used diagnosed NAFLD patients in comparison to our population without diagnosis of NAFLD [27]. Interestingly, the inverse association between PB-CML and LGI also disappeared after adjustment for BMI. Likely, this has to do with the aforementioned trapping of $\mathrm{PB}-\mathrm{CML}$ in the adipose tissue [13].

Of note, AGEs can form exogenously and are present in many food products [38]. The intestines absorb these dietary AGEs and we have recently demonstrated that a higher dietary AGE intake is associated with higher levels of free AGEs in plasma and urine [39]. These data support the idea that circulating free AGE levels are influenced by many factors including dietary AGE intake and as reported in the present study, liver health status.

Our study has some limitations. Given our crosssectional study design, we cannot draw causal conclusions on the investigated relationships. Prospective studies are required to better understand the contribution of liver disease to AGE formation and subsequently circulating AGEs and low-grade inflammation. Another important consideration is the estimation of liver fat employed in the study. We used two different equations to estimate liver fat content but did not measure liver fat content using imaging techniques or quantify hepatic steatosis or inflammation in liver biopsies. These procedures were not feasible in our large human cohort due to cost or ethical concerns. However, the two measures of liver fat (eLF\% and FLI) used in this study have been validated in previous studies and revealed similar associations in the present study $[19,20]$.

\section{Conclusions}

Our findings suggest an elevated formation of AGEs in fatty and injured livers accompanied with an enhanced breakdown of the formed protein-bound AGEs resulting in altered levels of circulating AGEs. Potentially, circulating AGEs can contribute to LGI.

\section{Abbreviations}

AGE: $\quad$ Advanced glycation end product

CEL:

CML:

CVD:
$\mathrm{N}^{\varepsilon}$-(1-carboxyethyl)lysine

$\mathrm{N}^{\varepsilon}$-(carboxymethyl)lysine

Cardiovascular disease 
LGI: Low-grade inflammation

MG-H1: $\quad \mathrm{N}^{\delta}$-(5-hydro-5-methyl-4-imidazolon-2-yl)ornithine

NAFLD: $\quad$ Nonalcoholic fatty liver disease

NASH: Nonalcoholic steatohepatitis

RAGE: $\quad$ Receptor for AGEs

TNF: $\quad$ Tumour necrosis factor

UPLC MS/MS: Ultraperformance liquid chromatography tandem mass spectrometry.

\section{Data Availability}

The datasets generated during and/or analysed during the current study are available from the corresponding author on reasonable request.

\section{Disclosure}

This work is based on a chapter in the dissertation of Mitchell Bijnen.

\section{Conflicts of Interest}

The authors declare that there is no conflict of interest regarding the publication of this paper.

\section{Authors' Contributions}

$\mathrm{MB}$ analysed the data and wrote the manuscript. MMJvG collected and analysed data and provided statistical advice. CJHvdK collected and analysed data. JLS designed methods and performed measurements. MPHvdW performed measurements. CDAS supervised analyses and revised the manuscript. KW designed the study, supervised data analyses, and revised the manuscript. CGS designed the study, supervised data analyses, and revised the manuscript.

\section{Acknowledgments}

The authors thank Ying Xin and Nynke Simons for providing statistical advice. This study was financed by the Netherlands Heart Foundation (2013T143) grant to Kristiaan Wouters. Initiation of CODAM was supported by grants of the Netherlands Organisation for Scientific Research (940-35-034) and the Dutch Diabetes Research Foundation (98.901).

\section{Supplementary Materials}

Suppl. Table 1: associations of FLI with free and proteinbound AGEs and sRAGE. $\beta$-Values are unstandardized regression coefficients and represent the change in AGEs and sRAGE (all ln-transformed) per one unit increase in FLI (standardized). Model 1: crude model. Model 2: model $1+$ adjustment for age and sex. Model 3: model $2+$ adjustment for alcohol, smoking, CVD, medication, and eGFR. Model 4: model 3 + adjustment for BMI. 95\% CI: 95\% confidence interval; FLI: fatty liver index. Suppl. Table 2: associations of FLI with low-grade inflammation. $\beta$-Values are unstandardized regression coefficients and represent the change in the LGI score per one unit increase in FLI (standardized). Model 1: crude model. Model 2: model 1 + adjustment for age and sex. Model 3: model $2+$ adjustment for alcohol, smoking, CVD, medication, and eGFR. Model 4: model 3+adjustment for BMI. 95\% CI: 95\% confidence interval; FLI: fatty liver index. (Supplementary Materials)

\section{References}

[1] A. Tailleux, K. Wouters, and B. Staels, "Roles of PPARs in NAFLD: potential therapeutic targets," Biochimica et Biophysica Acta, vol. 1821, no. 5, pp. 809-818, 2012.

[2] M. Ekstedt, L. E. Franzén, U. L. Mathiesen et al., "Long-term follow-up of patients with NAFLD and elevated liver enzymes," Hepatology, vol. 44, no. 4, pp. 865-873, 2006.

[3] D. Festi, A. Colecchia, T. Sacco, M. Bondi, E. Roda, and G. Marchesini, "Hepatic steatosis in obese patients: clinical aspects and prognostic significance," Obesity Reviews, vol. 5, no. 1, pp. 27-42, 2004.

[4] N. N. Than and P. N. Newsome, "A concise review of nonalcoholic fatty liver disease," Atherosclerosis, vol. 239, no. 1, pp. 192-202, 2015.

[5] P. Angulo, "Nonalcoholic fatty liver disease," The New England Journal of Medicine, vol. 346, no. 16, pp. 1221-1231, 2002.

[6] K. H. J. Gaens, P. M. G. Niessen, S. S. Rensen et al., "Endogenous formation of $N^{\varepsilon}$-(carboxymethyl)lysine is increased in fatty livers and induces inflammatory markers in an in vitro model of hepatic steatosis," Journal of Hepatology, vol. 56, no. 3, pp. 647-655, 2012.

[7] P. J. Thornalley, "Dicarbonyl intermediates in the maillard reaction," Annals of the New York Academy of Sciences, vol. 1043, no. 1, pp. 111-117, 2005.

[8] M. Brownlee, "Biochemistry and molecular cell biology of diabetic complications," Nature, vol. 414, no. 6865, pp. 813-820, 2001.

[9] E. S. Tai, M. L. S. Tan, R. D. Stevens et al., "Insulin resistance is associated with a metabolic profile of altered protein metabolism in Chinese and Asian-Indian men," Diabetologia, vol. 53, no. 4, pp. 757-767, 2010.

[10] A. Bierhaus, P. M. Humpert, M. Morcos et al., "Understanding RAGE, the receptor for advanced glycation end products," Journal of Molecular Medicine, vol. 83, no. 11, pp. 876-886, 2005.

[11] T. Kislinger, C. Fu, B. Huber et al., "N(epsilon)-(carboxymethyl)lysine adducts of proteins are ligands for receptor for advanced glycation end products that activate cell signaling pathways and modulate gene expression," The Journal of Biological Chemistry, vol. 274, no. 44, pp. 31740-31749, 1999.

[12] J. Xue, R. Ray, D. Singer et al., “The receptor for advanced glycation end products (RAGE) specifically recognizes methylglyoxal-derived AGEs," Biochemistry, vol. 53, no. 20, pp. 3327-3335, 2014.

[13] K. H. J. Gaens, G. H. Goossens, P. M. Niessen et al., "N${ }^{\varepsilon}$-(Carboxymethyl)lysine-receptor for advanced glycation end product axis is a key modulator of obesity-induced dysregulation of adipokine expression and insulin resistance," Arteriosclerosis, Thrombosis, and Vascular Biology, vol. 34, no. 6, pp. 1199-1208, 2014.

[14] B. I. Hudson, A. M. Carter, E. Harja et al., "Identification, classification, and expression of RAGE gene splice variants," The FASEB Journal, vol. 22, no. 5, pp. 1572-1580, 2008.

[15] A. Raucci, S. Cugusi, A. Antonelli et al., "A soluble form of the receptor for advanced glycation endproducts (RAGE) is 
produced by proteolytic cleavage of the membrane-bound form by the sheddase a disintegrin and metalloprotease 10 (ADAM10)," The FASEB Journal, vol. 22, no. 10, pp. 3716-3727, 2008.

[16] K. Nakamura, S. Yamagishi, H. Adachi et al., "Serum levels of sRAGE, the soluble form of receptor for advanced glycation end products, are associated with inflammatory markers in patients with type 2 diabetes," Molecular Medicine, vol. 13, no. 3-4, pp. 185-189, 2007.

[17] K. Nakamura, S. Yamagishi, H. Adachi et al., "Elevation of soluble form of receptor for advanced glycation end products (sRAGE) in diabetic subjects with coronary artery disease," Diabetes/Metabolism Research and Reviews, vol. 23, no. 5, pp. 368-371, 2007.

[18] M. Jacobs, M. M. J. van Greevenbroek, C. J. H. van der Kallen et al., "Low-grade inflammation can partly explain the association between the metabolic syndrome and either coronary artery disease or severity of peripheral arterial disease: the CODAM study," European Journal of Clinical Investigation, vol. 39, no. 6, pp. 437-444, 2009.

[19] A. Kotronen, M. Peltonen, A. Hakkarainen et al., "Prediction of non-alcoholic fatty liver disease and liver fat using metabolic and genetic factors," Gastroenterology, vol. 137, no. 3, pp. 865$872,2009$.

[20] G. Bedogni, S. Bellentani, L. Miglioli et al., "The fatty liver index: a simple and accurate predictor of hepatic steatosis in the general population," BMC Gastroenterology, vol. 6, no. 1, p. 33, 2006.

[21] D. J. Cuthbertson, M. O. Weickert, D. Lythgoe et al., "External validation of the fatty liver index and lipid accumulation product indices, using $1 \mathrm{H}$-magnetic resonance spectroscopy, to identify hepatic steatosis in healthy controls and obese, insulin-resistant individuals," European Journal of Endocrinology, vol. 171, no. 5, pp. 561-569, 2014.

[22] N. Wlazlo, M. M. J. van Greevenbroek, I. Ferreira et al., "Activated complement factor 3 is associated with liver fat and liver enzymes: the CODAM study," European Journal of Clinical Investigation, vol. 43, no. 7, pp. 679-688, 2013.

[23] N. M. J. Hanssen, L. Engelen, I. Ferreira et al., "Plasma levels of advanced glycation endproducts $\mathrm{N}^{\varepsilon}$-(carboxymethyl)lysine, $\mathrm{N}^{\varepsilon}$-(carboxyethyl)lysine, and pentosidine are not independently associated with cardiovascular disease in individuals with or without type 2 diabetes: the Hoorn and CODAM studies," The Journal of Clinical Endocrinology and Metabolism, vol. 98, no. 8, pp. E1369-E1373, 2013.

[24] Z. Yesilova, H. Yaman, C. Oktenli et al., "Systemic markers of lipid peroxidation and antioxidants in patients with nonalcoholic fatty liver disease," The American Journal of Gastroenterology, vol. 100, no. 4, pp. 850-855, 2005.

[25] M. Konishi, M. Iwasa, J. Araki et al., "Increased lipid peroxidation in patients with non-alcoholic fatty liver disease and chronic hepatitis $\mathrm{C}$ as measured by the plasma level of 8-isoprostane," Journal of Gastroenterology and Hepatology, vol. 21, no. 12, pp. 1821-1825, 2006.

[26] P. Lettéron, B. Fromenty, T. Benoît, C. Degott, and D. Pessayre, "Acute and chronic hepatic steatosis lead to in vivo lipid peroxidation in mice," Journal of Hepatology, vol. 24, no. 2, pp. 200-208, 1996.

[27] S. A. Palma-Duran, M. D. Kontogianni, A. Vlassopoulos et al., "Serum levels of advanced glycation end-products (AGEs) and the decoy soluble receptor for AGEs (sRAGE) can identify non-alcoholic fatty liver disease in age-, sex- and BMImatched normo-glycemic adults," Metabolism, vol. 83, pp. 120-127, 2018.

[28] M. R. Charlton, "Protein metabolism and liver disease," Baillière's Clinical Endocrinology and Metabolism, vol. 10, no. 4, pp. 617-635, 1996.

[29] M. Gaggini, F. Carli, C. Rosso et al., "Altered amino acid concentrations in NAFLD: impact of obesity and insulin resistance," Hepatology, vol. 67, no. 1, pp. 145-158, 2018.

[30] D. R. Sell and V. M. Monnier, "Structure elucidation of a senescence cross-link from human extracellular matrix. Implication of pentoses in the aging process," The Journal of Biological Chemistry, vol. 264, no. 36, pp. 21597-21602, 1989.

[31] I. D. Munsterman, T. J. Kendall, N. Khelil et al., "Extracellular matrix components indicate remodelling activity in different fibrosis stages of human non-alcoholic fatty liver disease," Histopathology, vol. 73, no. 4, pp. 612-621, 2018.

[32] I. Koborová, R. Gurecká, M. Csongová et al., “Association between metabolically healthy central obesity in women and levels of soluble receptor for advanced glycation end products, soluble vascular adhesion protein-1, and activity of semicarbazide-sensitive amine oxidase," Croatian Medical Journal, vol. 58, no. 2, pp. 106-116, 2017.

[33] M. Guclu, A. Ali, D. U. Eroglu, S. O. Büyükuysal, S. Cander, and N. Ocak, "Serum levels of sRAGE are associated with body measurements, but not glycemic parameters in patients with prediabetes," Metabolic Syndrome and Related Disorders, vol. 14, no. 1, pp. 33-39, 2016.

[34] K. Reyes-Gordillo, R. Shah, and P. Muriel, "Oxidative stress and inflammation in hepatic diseases: current and future therapy," Oxidative Medicine and Cellular Longevity, vol. 2017, Article ID 3140673, 2 pages, 2017.

[35] M. X. Fu, J. R. Requena, A. J. Jenkins, T. J. Lyons, J. W. Baynes, and S. R. Thorpe, "The advanced glycation end product, $\mathrm{N}^{\varepsilon}$-(carboxymethyl)lysine, is a product of both lipid peroxidation and glycoxidation reactions," Journal of Biological Chemistry, vol. 271, no. 17, pp. 9982-9986, 1996.

[36] J. W. Haukeland, J. K. Damås, Z. Konopski et al., "Systemic inflammation in nonalcoholic fatty liver disease is characterized by elevated levels of CCL2," Journal of Hepatology, vol. 44, no. 6, pp. 1167-1174, 2006.

[37] G. Tarantino, S. Savastano, and A. Colao, "Hepatic steatosis, low-grade chronic inflammation and hormone/growth factor/adipokine imbalance," World Journal of Gastroenterology, vol. 16, no. 38, pp. 4773-4783, 2010.

[38] J. L. J. M. Scheijen, E. Clevers, L. Engelen et al., "Analysis of advanced glycation endproducts in selected food items by ultra-performance liquid chromatography tandem mass spectrometry: presentation of a dietary AGE database," Food Chemistry, vol. 190, pp. 1145-1150, 2016.

[39] J. L. J. M. Scheijen, N. M. J. Hanssen, M. M. van Greevenbroek et al., "Dietary intake of advanced glycation endproducts is associated with higher levels of advanced glycation endproducts in plasma and urine: the CODAM study," Clinical Nutrition, vol. 37, no. 3, pp. 919-925, 2018. 


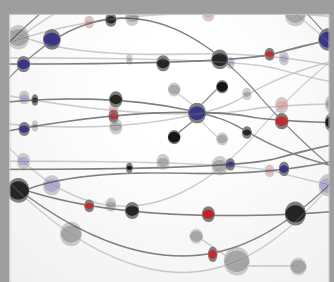

The Scientific World Journal
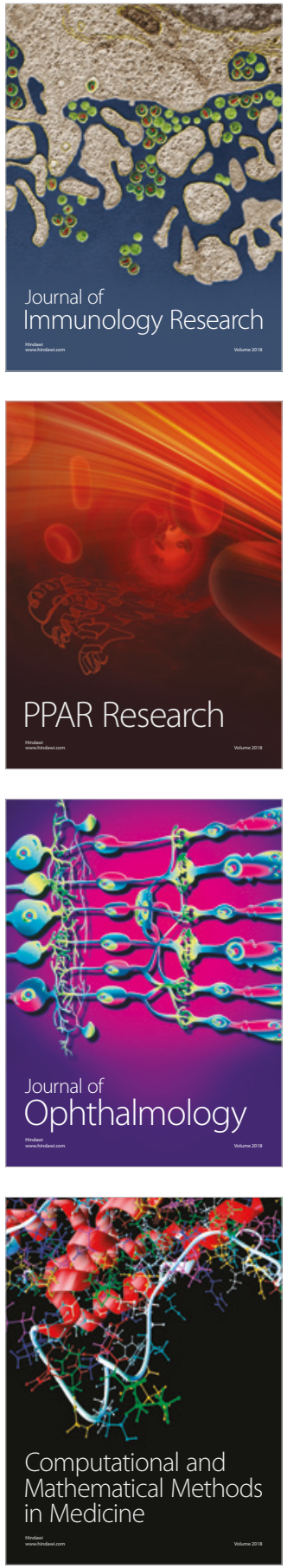

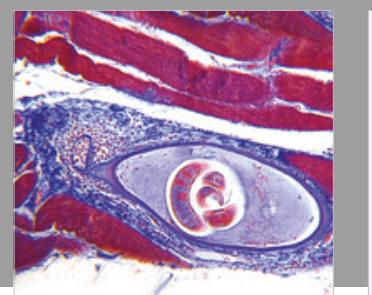

Gastroenterology Research and Practice

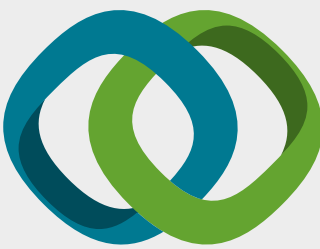

\section{Hindawi}

Submit your manuscripts at

www.hindawi.com
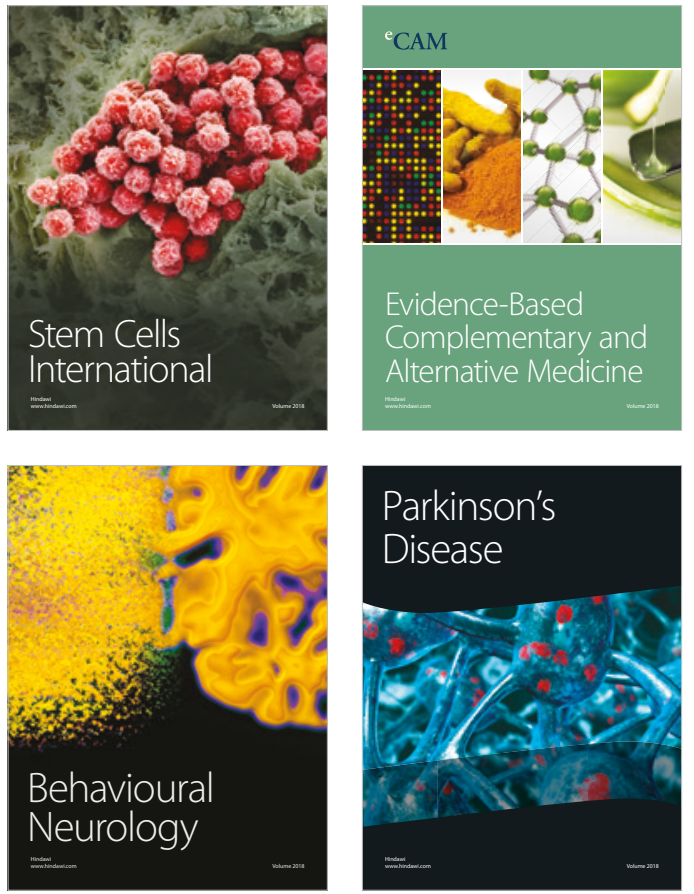

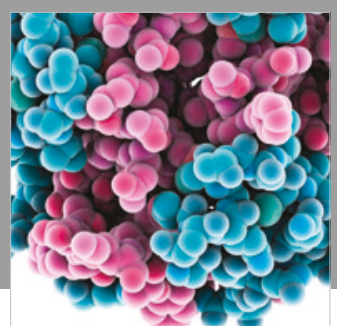

ournal of

Diabetes Research

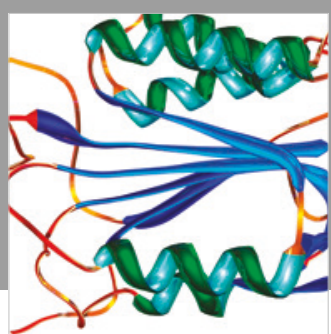

Disease Markers
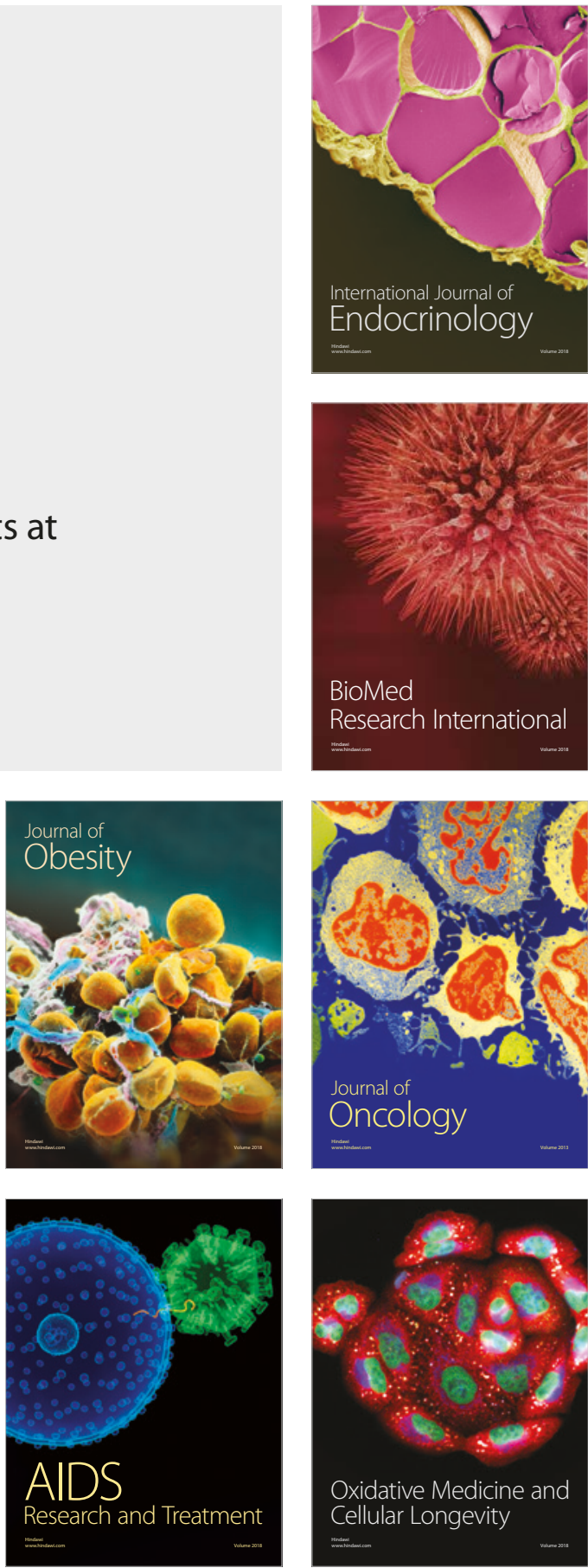